\title{
Competition and genotypic variability in Drosophila melanogaster
}

\author{
M. J. Martin,* \\ J. M. Pérez-Tomé* and \\ M. A. Toro $\dagger$
}

\author{
* Departamento de Genética, Facultad de Biología, \\ Ciudad Universitaria s/n., 28040 Madrid, Spain. \\ † Departamento de Genética Cuantitativa, \\ Instituto Nacional de Investigaciones Agrarias, \\ Ctra. de la Coruña Km. 7, 28034 Madrid, Spain.
}

It has been claimed that in competitive conditions larval viabilities of Drosophila melanogaster depend on the similarity of genotypes coexisting. Two types of experimental populations were established: homogeneous (low genotypic variability) and heterogeneous (high genotypic variability). Under conditions of moderate larval competition productivity was similar, in each although the developmental time was shorter in the heterogeneous series. Under conditions of high larval competition the productivity of heterogeneous populations exceeded that of the homogeneous and attained the adult stage in shorter period of time.

\section{INTRODUCTION}

It is a common assumption in the ecological literature that conspecifics compete more strongly than members of different species. An extension of this assumption is the idea that similar genotypes compete more than non-similar geneotypes because different genotypes utilise limited environmental resources, such as food, in different ways and therefore it follows that a mixture of various genotypes would exploit the resources better than a population that is genotypically uniform. If this kind of ecologically mediated frequency-dependent selection is a general one, it would be important as an explanation of the maintenance of genetic polymorphism and sexual reproduction systems (Tosic and Ayala, 1980; Antonovics and Ellstrand, 1984).

Experimental evidence supporting this point of view comes from competition experiments among Drosophild mutants (Lewontin and Matsuo, 1963; Caligari, 1980) and from plant agricultural experiments comparing the productivity of genotypic mixtures versus monocultures (Allard and Adams, 1969). Recently, Pérez-Tomé and Toro, (1982) claimed that competition of similar as opposed to non-similar geneotypes could be detected in a random mating population not subject to genetic manipulation. Vials with eggs laid by 10 unrelated females each mated to a different male (heterogeneous series) produced more surviving adults than vials with eggs laid by 10 unrelated females each one mated to the same male (homogeneous series). The only difference between both series is that in the first the full-sib families that compete are related as half-sibs whereas in the second these full-sib families are randomly assorted. However since the genotypes sampled in the two series are the same the authors interpreted their results as evidence for frequencydependent selection.

This interpretation has been queried by Fowler and Partridge (1986). They confirmed the above results but offered a different interpretation in terms of variation in male fertility. If such variation exists the between-vial variance of the number of fertile eggs produced would have been higher in the homogeneous series. If as a result of larval competition, survival rates declined with increasing larval density, the differences in variance would lead to differences in mean because the higher variance will be associated with low survival rates and hence lower productivity. They offered two lines of evidence supporting their claim. First, that variation in male fertility did indeed exist. Second, that differences in adult productivity between the series was abolished by standardisation of larval densities. The aim of the present work is to throw some light on this controversy. 


\section{MATERIALS AND METHODS}

The Drosophila melanogaster used was founded from a cross of 70 isofemale lines derived from flies collected by E. Torroja in Carboneras (Almería) in September 1981. Since then the population has been maintained on a standard food medium made with $100 \mathrm{~g}$ of yeast, $100 \mathrm{~g}$ of sucrose and $11 \mathrm{~g}$ of agar-agar per litre of water, adding $5 \mathrm{ml}$ of propionic acid after boiling. The flies were kept in an incubator room at $25 \pm 1.5^{\circ} \mathrm{C}$ under continuous illumination. All handling was at room temperature using ether anaesthesia.

\section{Experiment 1. Cultures at low density}

Virgin males and females were collected within 6 hours of emergence and aged for 3 days. The matings were set up placing each one of 28 males with 10 females in a vial for 96 hours, after which the male was removed. Two series of vials were then established. In the first, called the homogeneous series, the 10 females that had mated with the same male were placed in food-vials containing $1.5 \mathrm{ml}$ of standard medium. In the second, called the heterogeneous sereies, 10 females chosen randomly, but excluding any females mated with the same male, were placed in the same vial. All the females were transferred to fresh vials after 24 hours for a second egg-laying period. In both series, the medium of the vials was carefully removed after the laying period and placed in half pint milk bottles with $40 \mathrm{ml}$ of medium in order to avoid food competition.

\section{Experiment 2. Larval cultures with standard medium}

Mating groups of 1 male and 10 females, aged for 3 days, were set up in egg laying vials with removable bottom holding $1.5 \mathrm{ml}$ of standard medium blackened by the addition of $50 \mathrm{~g}$ per litre of powdered charcoal. The flies were transferred to fresh vials after 18 hours for a second laying period. The eggs were incubated during 24 hours and then the larvae were carefully collected and transferred to vials $(60 \times 17 \mathrm{~mm})$ containing $0.5 \mathrm{ml}$ of standard medium. The initial larval density was of 50 larvae per vial. In the homogeneous series the 50 larvae come from only one egg laying vial, whereas in the heterogeneous series the 50 larvae came from 10 egg laying vials, 5 per each one. The experiment was replicated twice in a three week interval.

\section{Experiment 3. Larval cultures with impoverished medium}

There are three ways of increasing the competition among larvae in the vials. The first is to increase the number of larvae per culture. This method is difficult to carry out because in the homogeneous series all the larvae must come from the same mating group. The second is to reduce the amount of food, but amounts of food lower than $0.5 \mathrm{ml}$ are very susceptible to changes in the degree of humidity and in many vials all larvae could be killed by drying. The third method is to impoverish the food medium and it was the method chosen in this experiment. The amount of yeast was reduced by half $(50 \mathrm{~g} / 1)$. Preliminary results indicated that the impoverished medium was very sensitive to slight microenvironmental changes that provoked differences in the degree of desiccation of vials. In order to reduce the differences in humidity among vials the impoverished food medium was added over $1 \mathrm{ml}$ of previously solidified agar-agar solution $(11 \mathrm{~g} / 1)$, that buffered the environmental fluctuations although they did not disappear completely. The method of handling and the number of transferred larvae was identical to experiment 2. This experiment was replicated three times scheduled with delays of 3 weeks.

\section{RESULTS}

\section{Experiment 1. Cultures at low density}

The results are given in table 1 . There is a considerable decrease in mean adult productivity in the

Table 1 Means and variances of adult productivity in the Heterogeneous and Homogeneous series (Experiment 1)

\begin{tabular}{|c|c|c|c|c|c|c|c|c|}
\hline \multirow[b]{2}{*}{ Period } & \multicolumn{3}{|c|}{ Heterogeneous } & \multicolumn{3}{|c|}{ Homogeneous } & \multirow[b]{2}{*}{$t$} & \multirow[b]{2}{*}{$F$} \\
\hline & $n$ & $\bar{x}$ & $V$ & $n$ & $\bar{x}$ & $V$ & & \\
\hline 1 & 28 & $110 \cdot 75$ & $835 \cdot 53$ & 28 & $115 \cdot 57$ & $2217 \cdot 89$ & 0.46 & $2 \cdot 65^{* *}$ \\
\hline 2 & 28 & $43 \cdot 14$ & $206 \cdot 41$ & 28 & $42 \cdot 96$ & $506 \cdot 32$ & 0.03 & $2 \cdot 45^{*}$ \\
\hline
\end{tabular}

${ }^{*} p<0.05,{ }^{* *} p<0.01$ 
Table 2 Means of adult productivity and mean developmental times in the heterogeneous and homogeneous series (Experiment 2)

\begin{tabular}{|c|c|c|c|c|c|c|}
\hline \multirow[b]{2}{*}{ Replicate } & \multicolumn{2}{|c|}{ Heterogeneous } & \multicolumn{2}{|c|}{ Homogeneous } & \multirow[b]{2}{*}{$t$} & \multirow[b]{2}{*}{$F$} \\
\hline & $n$ & $\bar{x} \pm$ S.E. & $n$ & $\bar{x} \pm$ S.E. & & \\
\hline \multicolumn{7}{|c|}{ Adult productivity } \\
\hline 1 & 28 & $35 \cdot 75 \pm 0 \cdot 96$ & 23 & $35 \cdot 60 \pm 0 \cdot 97$ & $0 \cdot 11$ & $1 \cdot 19$ \\
\hline 2 & 10 & $34 \cdot 10 \pm 0 \cdot 82$ & 7 & $37 \cdot 00 \pm 2 \cdot 19$ & $1 \cdot 24$ & $4.99^{*}$ \\
\hline \multicolumn{7}{|c|}{ Developmental time (days) } \\
\hline 1 & 1001 & $13 \cdot 53 \pm 0 \cdot 04$ & 819 & $14 \cdot 29 \pm 0 \cdot 05$ & $11 \cdot 86^{* * *}$ & \\
\hline 2 & 341 & $14 \cdot 17 \pm 0 \cdot 11$ & 259 & $15 \cdot 18 \pm 0 \cdot 10$ & $6.93^{* * *}$ & \\
\hline
\end{tabular}

${ }^{*} p<0 \cdot 05,{ }^{* * *} p<0.001$

second laying period that can be explained by the depletion of the sperm accumulated by the females in the previous mating period. The small differences in productivity between the two series were not significant, However, for each period, the variance of productivity was greater in the homogeneous series and the difference was significant. All adults emerged almost simultaneously with developmental times of 11 or 12 days.

\section{Experiment 2. Larval cultures with standard medium}

Two egg-laying periods were evaluated in replicate 1 but only one in replicate 2 . The number of vials was always lesser in the homogeneous series due to the difficulty of obtaining the required 50 larvae coming from a single mating group. Because there were no differences between periods the data were pooled in replicate 1 . The mean productivity and the mean developmental time are given in table 2 for the two series. There were no significant differences with respect to adult productivity but despite the larval standarisation the variance of the productivity was greater in the homogeneous series and the mean developmental time, measured in days, was in all cases significantly shorter in the heterogeneous series. Fig. 1 shows the daily mean productivity for the two series reflecting the same phenomenon.

\section{Experiment 3. Larval culture with impoverished medium}

For each replicate the results were pooled over the two periods because they were not significantly different and are summarised in table 3 . In the first replicate the viability from larva to adult was about 50 per cent and the adult productivity was significantly greater in the heterogeneous series. The developmental time was also shorter in this series. In the second and third replicate there is a strong

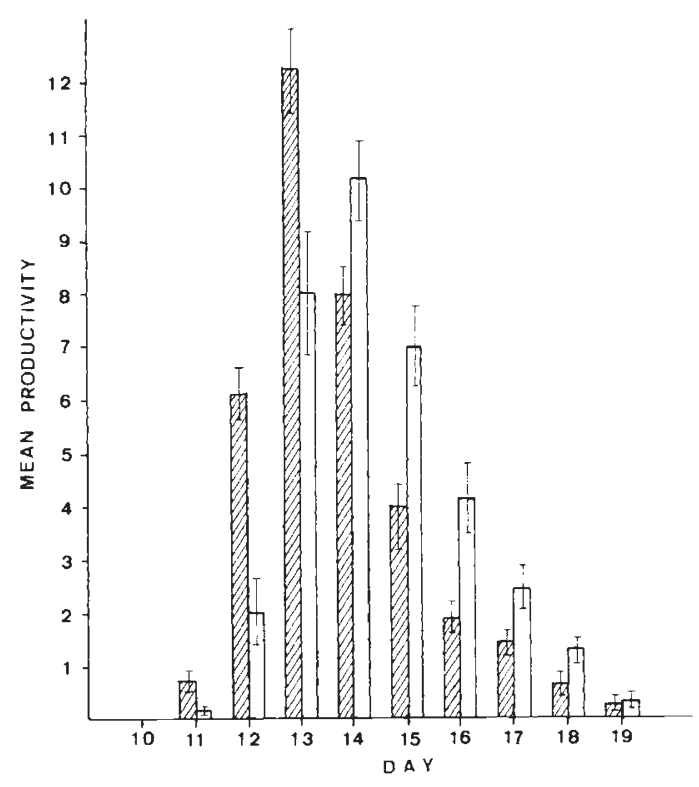

Figure 1 Mean number of flies of replicate 1 and 2 emerging daily for the Heterogeneous (绍) and Homogeneous ( $\square$ ) series.

decrease in viability down to values of 25 per cent or less and the developmental times were greater than in the first replicate. This suggests that the competition level has been lower in this replicate probably because the amount of food or the proportion of yeast in it was, by mistake, greater than intended. The difference between the homogeneous and the heterogeneous series was larger in replicate 3 than in replicate 2 probably due to environmental causes that increased the level of competition in replicate 3 .

In any case, for each replicate the mean productivity of the heterogeneous series exceeded that of the homogeneous series the difference being highly significant in two of them. For all replicates the variance of productivity was significantly greater 
Table 3 Means of adult productivity and mean developmental times in the heterogeneous and homogeneous series (Experiment 3)

\begin{tabular}{|c|c|c|c|c|c|c|}
\hline \multirow[b]{2}{*}{ Replicate } & \multicolumn{2}{|c|}{ Heterogeneous } & \multicolumn{2}{|c|}{ Homogeneous } & \multirow[b]{2}{*}{$t$} & \multirow[b]{2}{*}{$F$} \\
\hline & $n$ & $\bar{x} \pm$ S.E. & $n$ & $\bar{x} \pm$ S.E. & & \\
\hline \multicolumn{7}{|c|}{ Adult productivity } \\
\hline 1 & 60 & $27 \cdot 28 \pm 0 \cdot 48$ & 47 & $23.02 \pm 0.73$ & $4 \cdot 88^{* * *}$ & $1 \cdot 81^{*}$ \\
\hline 2 & 30 & $11.67 \pm 0.48$ & 30 & $10 \cdot 37 \pm 0 \cdot 70$ & $1 \cdot 53$ & $2 \cdot 13^{*}$ \\
\hline 3 & 88 & $11 \cdot 39 \pm 0.50$ & 54 & $6 \cdot 44 \pm 0 \cdot 76$ & $5 \cdot 42 * * *$ & $1 \cdot 42$ \\
\hline \multicolumn{7}{|c|}{ Developmental time (Days) } \\
\hline 1 & 1640 & $13.64 \pm 0.08$ & 1082 & $14 \cdot 35 \pm 0 \cdot 11$ & $5 \cdot 22^{* * *}$ & \\
\hline 2 & 350 & $16 \cdot 69 \pm 0 \cdot 21$ & 311 & $17 \cdot 60 \pm 0 \cdot 23$ & $2 \cdot 88^{* *}$ & \\
\hline 3 & 1003 & $21 \cdot 60 \pm 0 \cdot 17$ & 348 & $22 \cdot 75 \pm 0 \cdot 31$ & $3 \cdot 20^{* *}$ & \\
\hline
\end{tabular}

${ }^{*} p<0.05,{ }^{* *} p<0.01,{ }^{* * *} p<0.001$

in the homogeneous series. As in experiment 2, the larvae of the heterogeneous series always attained the adult stage in a shorter period of time, the differences being always significant.

\section{DISCUSSION}

The results of Pérez-Tomé and Toro (1982), showing that in competitive conditions larval viabilities of Drosophila melanogaster depend on the similarity of the geneotypes coexisting, can be partially explained, as proposed by Fowler and Partridge (1986), in terms of variation in male fertility. The results of Experiment 1, where cultures were set up at low density and presumably with minimum competition, show that the mean productivity of both series was equal although the variance in the number of eggs laid in the variance in the homogeneous series was more than two times that of heterogeneous series. In Experiment 2 we set up cultures at density of 50 larvae per $0.5 \mathrm{ml}$ of standard food medium and, as in the results of Fowler and Partridge (1986), we did not find differences in mean adult productivity between series. However, developmental time was shorter in the heterogeneous series which seems to imply that conditions for development are more favourable in the cultures with higher genotypic variability.

In both, our Experiment 2 and that reported by Fowler and Partridge (1986) the observed viabilities can hardly indicate conditions as being highly competitive since in most competition studies viabilities fall below 30 per cent (Moya and Botella, 1985). Under conditions of moderate competition only differences in development time between series can be detected. If the intensity of competition is increased, reducing the amount of yeast in the food by half (Experiment 3 ) the advantage of the heterogeneous series is clearly manifested in greater productivity. The results with respect to the developmental time also support the hypothesis since the absolute differences between the homogeneous and the heterogeneous series were greater in replicate 2 and 3 than in replicate 1 although here the level of significance is superior due to the higher number of evaluated individuals. Furthermore, the vials with less geneotypic heterogeneity seems to be more sensible to microenvironmental variables as it is reflected in the larger variance of the number of adults produced.

Evidence of frequency-dependent selection has been accumulating in recent years and its implication in the maintenance of genetic polymorphism have been widely discussed (Snyder and Ayala, 1979; Tosic and Ayala, 1980). An ecological mechanism that creates a negative correlation between the frequency of genotypes and their fitness is that different genotypes utilise environmental resources in different ways. We have shown that this mechanism can work in highly competitive laboratory conditions although its relevance in natural conditions can be questioned. It would be most interesting if the fact that the fitness of genetically variable progeny being significantly greater than that of genetically uniform progeny was a general phenomenon in nature. This could provide a mechanism for the maintenance of sex through sib-competition (Maynard Smith, 1978; Price and Wasser, 1982; Ellstrand and Antonovics, 1985).

Acknowledgement This work was partially supported by a grant of the Comisión Asesora de Investigación Científicar y Técnica (CAICYT n. 2306/83).

\section{REFERENCES}

Allard, R. W. AND ADAMS, J. 1969. Population studies in predominantly self-pollinating species. XIII. Intergenotypic competition and population structure in Barley and wheat. Amer. Natur. 103, 621-645. 
ANTONOViCS, J. AND ELlSTRAND, N. C. 1984. Experimental studies of the evolutionary significance of sexual reproduction. I. A test of the frequency-dependent selection hypothesis. Evol., 38, 103-115.

CAlligari, P. D. S. 1980. Competitive interactions in Drosophila melanogaster. I. Monocultures. Heredity, 45, 219-231.

ELLSTRAND, N. C. AND ANTONOVICS, J. 1985. Experimental studies of the evolutionary significance of sexual reproduction. II. A test of the density-dependent selection hypothesis. Evol., 39, 657-666.

FOWLER, K. AND PARTRIDGE, L. 1986. Variation in male fertility explains an apparent effect of genotypic diversity on success in larval competition in Drosophila melanogaster. Heredity, 57, 31-36.

LEWONTIN, R. C. AND MATSUO, Y. 1963. Interaction of genotypes determining viability in Drosophila busckii. Proc. Natl. Acad. Sci., USA, 49, 270-278.
MAYNARD SMITH, J. 1978. The Evolution of Sex. Cambridge University Press. Cambridge.

MOYA, A. AND BOTELLA, L. M. 1985. Larva-to-adult and pupato-adult mortality dynamics in crowded cultures of Drosophila. Geneticü, 66, 201-207.

PÉREZ-TOMÉ, J. M. AND TORO, M. A. 1982. Competition of similar and non-similar genotypes. Nature, 299, 153-154.

PRICE, M. V. AND WASER, N. M. 1982. Population structure, frequency-dependent selection, and the maintenance of sexual reproduction. Evol., 36, 35-43.

SNYDER, T. P. AND AYALA, F. J. 1979. Frequency-dependent selection at the PGH-1 locus of Drosophila pseudoobscura. Genetics, 92, 995-1003.

TOSIC, M. AND AYALA, F. J. 1980. "Overcompensation" at an enzyme locus in Drosophila pseudoobscura. Genet. Res. Camb., 36, 57-67. 